University of Nebraska - Lincoln

DigitalCommons@University of Nebraska - Lincoln

4-26-2005

\title{
Search for global minimum geometries for medium sized germanium clusters: $\mathrm{Ge}_{12}-\mathrm{Ge}_{20}$
}

\author{
Satya S. Bulusu \\ University of Nebraska-Lincoln, sbulusu@iiti.ac.in \\ S. Yoo \\ University of Nebraska-Lincoln \\ Xiao Cheng Zeng \\ University of Nebraska-Lincoln, xzeng1@unl.edu
}

Follow this and additional works at: https://digitalcommons.unl.edu/chemzeng

Part of the Chemistry Commons

Bulusu, Satya S.; Yoo, S.; and Zeng, Xiao Cheng, "Search for global minimum geometries for medium sized germanium clusters: $\mathrm{Ge}_{12}-\mathrm{Ge}_{20}$ " (2005). Xiao Cheng Zeng Publications. 22.

https://digitalcommons.unl.edu/chemzeng/22

This Article is brought to you for free and open access by the Published Research - Department of Chemistry at DigitalCommons@University of Nebraska - Lincoln. It has been accepted for inclusion in Xiao Cheng Zeng Publications by an authorized administrator of DigitalCommons@University of Nebraska - Lincoln. 


\title{
Search for global minimum geometries for medium sized germanium clusters: $\mathrm{Ge}_{\mathbf{1 2}}-\mathrm{Ge}_{\mathbf{2 0}}$
}

\author{
S. Bulusu, S. Yoo, and X. C. Zeng ${ }^{\text {a) }}$ \\ Department of Chemistry and Center for Materials Research \& Analysis, University of Nebraska-Lincoln, \\ Lincoln, Nebraska 68588
}

(Received 16 December 2004; accepted 8 February 2005; published online 26 April 2005)

\begin{abstract}
We have performed an unbiased search for the global minimum geometries of small-to-medium sized germanium clusters $\mathrm{Ge}_{n}(12 \leqslant n \leqslant 18)$ as well as a biased search (using seeding method) for $\mathrm{Ge}_{n}(17 \leqslant n \leqslant 20)$. We employed the basin-hopping algorithm coupled with the plane-wave pseudopotential density functional calculations. For each size, we started the unbiased search with using several structurally very different initial clusters, or we started the biased search with three different seeds. Irrespective of the initial structures of clusters we found that the obtained lowest-energy clusters of the size $n=12-16$ and 18 are the same. Among them, the predicted global minima of $\mathrm{Ge}_{n}(12 \leqslant n \leqslant 16)$ are identical to those reported previously [Shvartsburg et al., Phys. Rev. Lett. 83, 167 (1999)]. For $n=17-20$, we have identified two or three nearly isoenergetic low-lying isomers (for each size) that compete for the global minimum. Nearly all the low-lying clusters in the size range of $12 \leqslant n \leqslant 20$ contain the tri-caped trigonal prism motif and are all prolate in geometry, in agreement with the experiment. (C) 2005 American Institute of Physics.
\end{abstract}

[DOI: $10.1063 / 1.1883647]$

\section{INTRODUCTION}

Study of growth patterns of small-to-medium sized and low-energy clusters can provide insight into evolution of matter from atom to microparticles and eventually to bulk solid. Over the past two decades semiconductor clusters have received considerable experimental interest ${ }^{1-12}$ largely because of their potential industrial applications. It is well known that at the surface of bulk silicon or germanium extensive reconstruction commonly occurs to minimize the number of surface dangling bonds. Thus, to achieve better understanding of properties of silicon or germanium microparticles it is of both fundamental and practical interests to understand the structures and properties of small-to-medium sized clusters. In particular, knowledge of geometric structures of low-lying clusters is important to the understanding of structural evolution and change in electronic properties as the size of clusters grows. Since the late 1990s the search for the global minima as well as their growth patterns for medium-sized silicon clusters $\operatorname{Si}_{n}(n \geqslant 12)$ has received much theoretical attention. ${ }^{13-31}$ It has been predicted that the global minima of $\mathrm{Si}_{10}-\mathrm{Si}_{15}$ (except $\mathrm{Si}_{14}$ ) contain the tri-capped trigonal prism (TTP) motif. ${ }^{13}$ A motif transition from TTP to the so-called six/six structural motif (referring to the sixfold puckered ring $\mathrm{Si}_{6}$ plus a tetragonal bipyramid $\mathrm{Si}_{6}$ complex) occurs at $n=16$, and another motif transition to the six/ten motif (referring to the sixfold puckered ring $\mathrm{Si}_{6}$ plus a tenatom magic-number cluster $\mathrm{Si}_{10}$ complex) occurs at $n=23 .{ }^{26,29,31}$ However, theoretical studies of the low-lying structures of germanium clusters are much less reported, ${ }^{32-36}$ especially for the medium-sized clusters $\mathrm{Ge}_{n}$ (e.g., $n \geqslant 12) .{ }^{37,38}$ Curtiss et al. ${ }^{33}$ calculated binding energies of

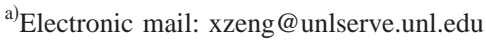

several isomers of $\mathrm{Ge}_{5}$ at $\mathrm{B} 3 \mathrm{LYP} / 6-311+\mathrm{G}(3 d f, 2 p)$ level of density-functional theory (DFT), which show reasonable agreement with the experimental data. Amant and Archibong $^{34}$ reported structures and electron affinities of small neutral and anionic clusters (up to $n=6$ ) using B3LYP level of DFT as well as coupled-cluster method with singles, doubles, and noniterative perturbative triples $[\operatorname{CCSD}(\mathrm{T})]$ in order to correlate their calculations with the photoelectron spectroscopy measurement. $\mathrm{Li}$ and $\mathrm{Cao}^{35}$ investigated smallsized low-lying clusters $(n=3-10)$ in more detail, using a full potential linear muffin-tin orbital molecular-dynamics method. They compared geometric structures of germanium with the silicon counterparts and found that the globalminimum geometries of the small germanium clusters are almost identical to those of silicon (except a few localminimum geometries). They also reported that the average bond-length in the germanium clusters is about $6 \%$ longer than the silicon counterparts. Ho and co-workers ${ }^{36}$ performed, perhaps, the first unbiased search for the global minima of germanium clusters. They combined CarParrinello molecular dynamics simulation with the simulated-annealing method and located the globalminimum clusters of $\mathrm{Si}_{n}, \mathrm{Ge}_{n}$, and $\mathrm{Sn}_{n}(n \leqslant 13)$. All these theoretical studies showed that the small-sized germanium clusters $(n \leqslant 11)$ have spherical-like compact geometries.

For $\mathrm{Ge}_{n}(n>13)$, we are aware of only two theoretical papers $^{37,38}$ on study of low-lying geometric structures. Ho and co-workers ${ }^{37}$ reported the lowest-energy structures of $\mathrm{Ge}_{n}$ up to $n=16$, by combining genetic algorithm (GA) with tight-binding method. Later, Wang et al. ${ }^{38}$ reported the lowest-energy geometries of small-to-medium sized clusters $(n=2-25)$, on the basis of GA combined with nonorthogonal-tight-binding method. They found that for 
$\mathrm{Ge}_{n}(n>13)$ both the stacked layered structures and the spherical-like compact structures compete for the lowestenergy structures.

On the experimental side, ion mobility measurements ${ }^{10}$ have revealed that the medium-sized clusters $\mathrm{Ge}_{n}$ are generally prolate in shape and the structural transition from the prolate to spherical-like shape appears at $n \sim 65$. Despite the many advances in experimental characterization of clusters over the two past decades, detailed morphology for most medium-sized clusters cannot be determined solely from experiments. Hence, determination of cluster structures has mainly relied on DFT and ab initio quantum-mechanical calculations. It is well known that as the size of clusters increases the number of local minima increases rapidly and so does the computational time required for the unbiased global search, particularly when the global search is combined with DFT or $a b$ initio calculations. In light of the fact that global minima of germanium clusters predicted previously were mostly based on semiempirical tight-binding calculations, the purpose of this study is to reexamine the global minima of $\mathrm{Ge}_{n}$ in the size range $12 \leqslant n \leqslant 20$ by using the basinhopping $(\mathrm{BH})$ global optimization technique $\mathrm{e}^{39,40}$ combined with the plane-wave pseudopotential DFT method. ${ }^{41-43} \mathrm{We}$ have recently employed this combined BH-DFT approach to locate a new global minimum of $\mathrm{Si}_{16} \cdot{ }^{31}$

\section{METHODS}

For the small-to-medium-sized germanium clusters $\mathrm{Ge}_{12}-\mathrm{Ge}_{20}$, we employed the $\mathrm{BH}$ global optimization technique combined with $\mathrm{DFT}^{30,31}$ to search for the globalminimum structures. The BH method essentially converts the potential energy surface $(\widetilde{E})$ to a multidimensional "staircase" via the mapping $\widetilde{E}(\mathbf{X})=\min \{E(\mathbf{X})\}$, where $\mathbf{X}$ denotes the nuclear coordinates of the cluster and "min" refers to the energy minimization performed starting from $\mathbf{X}$. In practice, the canonical Monte Carlo (MC) sampling method was used to explore the transformed $\widetilde{E}$ at a constant temperature. For each MC move, coordinates of all atoms are randomly displaced, followed by a geometry optimization using DFT. Specifically, the plane-wave pseudopotential DFT with gradient-corrected PBE functional (which is implemented in the CPMD program ${ }^{42}$ ) was adopted for the structural optimization. For each given cluster size, two to three independent $\mathrm{BH}$ searches were undertaken starting with very different initial cluster geometries. Typically, one initial structure is randomly generated, the second one is identical to a lowenergy silicon cluster with the same size, and the third is a flat planar structure. Obviously, the latter structure is highly unrealistic for germanium.

Two types of BH searches were performed, unbiased or biased search with seeding method. For larger clusters both unbiased and biased searches were used. In the first series, we carried out an unbiased global search for clusters $\mathrm{Ge}_{n}(12 \leqslant n \leqslant 18)$ using the BH-DFT method. For $12 \leqslant n$ $\leqslant 16$ and $n=18$, we found that despite marked differences among initial cluster structures, the BH-DFT search consistently yields identical lowest-energy isomer, typically, within 200-1000 MC trial moves. In Fig. 1, for example, we dis-

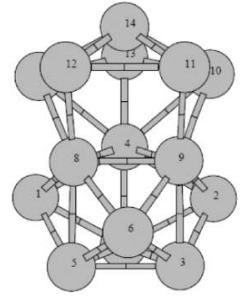

15a $\left(C_{s}\right)$

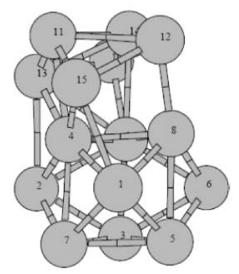

$15 \mathrm{c}\left(\mathrm{C}_{1}\right)$



$15 b\left(C_{3 v}\right)$

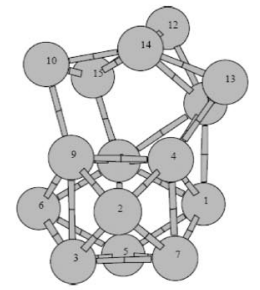

$15 d\left(C_{1}\right)$

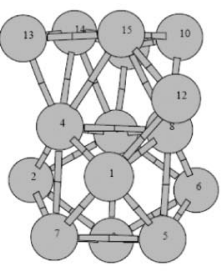

$15 \mathrm{e}\left(\mathrm{C}_{1}\right)$

FIG. 1. Geometries of the top-five most stable isomers of $\mathrm{Ge}_{15}$ based on three independent unbiased global searches using the combined basinhopping/density-functional theory method.

played top-five most stable isomers of $\mathrm{Ge}_{15}$, resulting from three independent BH/DFT searches with three different initial structures, respectively.

For larger clusters, however, the unbiased search becomes increasingly demanding in computing time. It is known that the number of local minima increases dramatically with the size of clusters. In the case of $\mathrm{Ge}_{17}$, for example, we found that the lowest-energy structures obtained via the $\mathrm{BH}$ searches were not always the same, but depending on the initial cluster structures, at least within $1000 \mathrm{MC}$ trial moves. Much larger number of MC moves (e.g., an order of magnitude larger) may solve this problem but would demand considerably more computing resources which are not yet available in our laboratory. We therefore performed a biased (but more efficient) search with seeding method for the four larger germanium clusters $\mathrm{Ge}_{n}(17 \leqslant n \leqslant 20)$. This approach has been used previously for silicon clusters. ${ }^{31} \mathrm{Ba}-$ sically, in the seeding approach, a structural motif is used as the seed. During the BH search, the atoms in the seed never undergo any MC trial moves, namely, only those atoms not included in the seed are allowed to undergo the MC trial moves. Typically, a good candidate of structural motif involves one or more magic-number semiconductor clusters, or some generic structures appearing in several low-lying clusters. Here, we have considered three structural motifs as a seed for the biased search. The first one is the TTP motif 


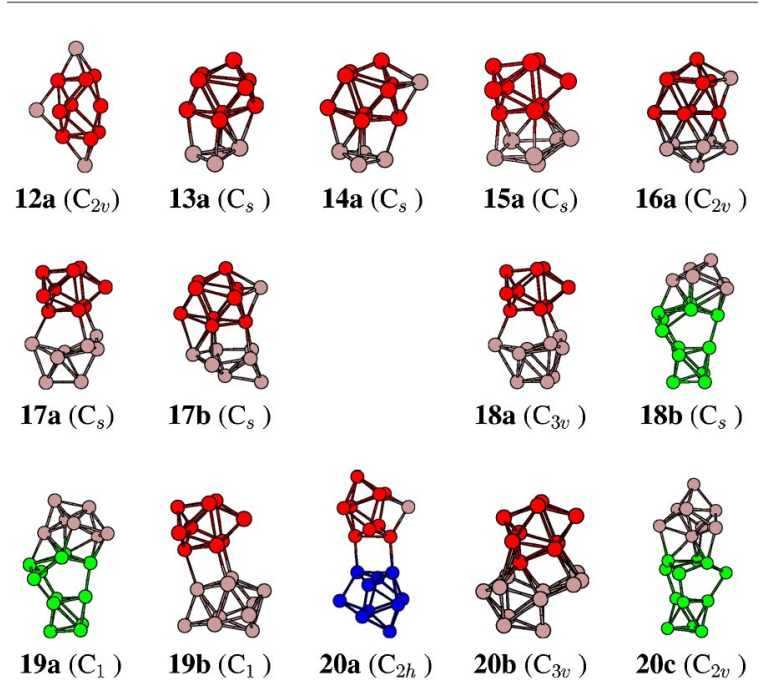

FIG. 2. (Color online) Geometries of the low-lying isomer structures of $\mathrm{Ge}_{12}-\mathrm{Ge}_{20}$ optimized at the PBEPBE/6-311G $(d)$ level of DFT. The TTP, six/six, and magic-number cluster $\mathrm{Ge}_{10}$ are highlighted by the red, green, and blue colors, respectively.

(highlighted via red color in Fig. 2), which is known to show in all the small low-lying isomers of $\mathrm{Ge}_{n}$. The second structural motif is the so-called six/six motif (highlighted via green color in Fig. 2) which refers to six-fold puckered ring $\mathrm{Ge}_{6}$ plus the tetragonal bipyramid $\mathrm{Ge}_{6}$ complex. Note that the latter $\mathrm{Ge}_{6}$ subunit is a magic-number cluster whereas the former $\mathrm{Ge}_{6}$ subunit is a part of "adamantane" unit, namely, a fragment of bulk diamond. The third structural motif is the magic-number cluster $\mathrm{Ge}_{10}$ (highlighted by the blue color in Fig. 2). Once the top-five most stable isomers were obtained, either from the unbiased or biased search, geometric optimi- zations were subsequently performed using (all-electron) PBEPBE method of DFT with the 6-311G $(d)$ basis set, which is implemented in the GAUSSIAN 03 software package. ${ }^{44}$ Vibrational analysis was also taken for all the optimized clusters to make sure the absence of imaginary frequencies. Geometry optimizations were also done with another popular hybrid exchange-correlation functional (B3LYP) with the same basis set. The purpose of this calculation is to rule out possibility of having different energy orders given by different DFT methods (PBE or B3LYP).

\section{RESULTS AND DISCUSSION}

The predicted global-minimum structures for the smallto-medium sized clusters $\mathrm{Ge}_{n}(n=12-20)$ are shown in Fig. 2. Figure 3 shows the global minima of the corresponding silicon structures, ${ }^{31}$ previously predicted with the same BHDFT approach. The single-point energies (in hartree) calculated for the low-lying isomers of $\mathrm{Ge}_{n}(n=17-20)$ with both the PBEPBE/6-311G $(d)$ and B3LYP/6-311G $(d)$ methods are listed in Table I. The binding (or cohesive) energies per atom (in $\mathrm{eV}$ ) along with the corresponding experimental values (Ref. 10) are given in Table II. The zero-point energy correction has been taken into account while evaluating the binding energies per atom. Note that the binding energies per atom increase as increasing the size of the germanium clusters. The binding energies calculated for all the lowestenergy clusters are in fair agreement with the corresponding experimental values. The discrepancy between the theory and the experiment is less than or about $0.15 \mathrm{eV}$. Note that the measured values are derived from dissociation data on cluster cations, combined with measured ionization energies.

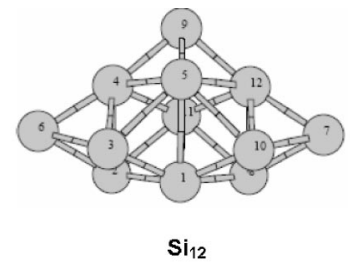

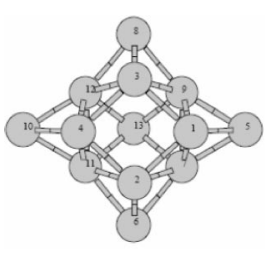

$\mathrm{Si}_{13}$

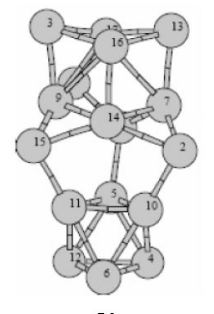

$\mathrm{Si}_{17}$

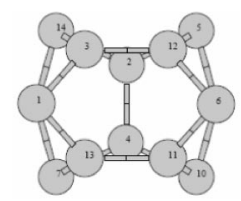

$\mathrm{Si}_{14}$

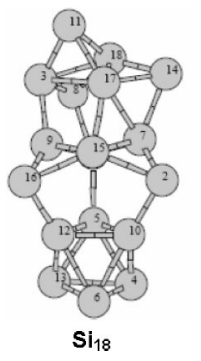

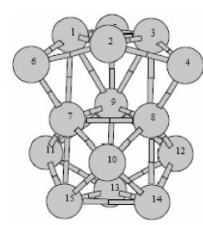

$\mathrm{Si}_{15}$

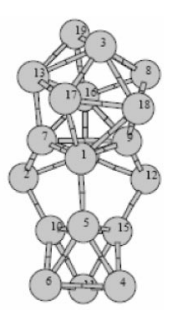

$\mathrm{Si}_{19}$

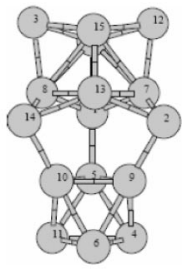

$\mathrm{Si}_{16}$

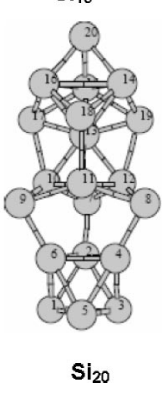

FIG. 3. Geometries of the predicted global-minimum geometries of medium-sized silicon clusters $\mathrm{Si}_{12}-\mathrm{Si}_{20}$ (Ref. 31). 
TABLE I. The single-point energies calculated at both PBEPBE/6 $-311 \mathrm{G}(d)$ and B3LYP/6-311G $(d)$ levels of DFT for the low-lying isomers of $\mathrm{Ge}_{17}-\mathrm{Ge}_{20}$ (shown in Fig. 2).

\begin{tabular}{|c|c|c|c|}
\hline Clusters & $\begin{array}{l}\text { Point } \\
\text { group }\end{array}$ & $\begin{array}{c}\mathrm{PBEPBE} / 6-311 \mathrm{G}(d) \\
\text { (hartree) }\end{array}$ & $\begin{array}{c}\text { B3LYP/6-311G }(d) \\
\text { (hartree) }\end{array}$ \\
\hline $17 \mathbf{a}$ & $C_{s}$ & -35303.7161134 & -35309.6032300 \\
\hline $17 \mathrm{~b}$ & $C_{s}$ & -35303.7138455 & -35309.5950869 \\
\hline $18 \mathbf{a}$ & $C_{3 v}$ & -37380.4064311 & -37386.6344676 \\
\hline $18 \mathrm{~b}$ & $C_{s}$ & -37380.3963262 & -37386.6319693 \\
\hline 19a & $C_{1}$ & -39457.1018824 & -39463.6839347 \\
\hline $19 b$ & $C_{1}$ & -39457.0982949 & -39463.6741134 \\
\hline $20 a$ & $C_{2 h}$ & -41533.8024729 & -41540.7294643 \\
\hline $20 \mathrm{~b}$ & $C_{3 v}$ & -41533.7934086 & -41540.7250944 \\
\hline $20 \mathrm{c}$ & $C_{2 v}$ & -41533.7903838 & -41540.6936330 \\
\hline
\end{tabular}

\section{A. $\mathrm{Ge}_{12}-\mathrm{Ge}_{16}$}

The global-minimum structures 12a-16a, obtained based on the unbiased search with the BH-DFT approach, are identical to those reported previously by Ho and co-workers. ${ }^{36,37}$ It can be seen that the TTP motif (highlighted in red color in Fig. 2) is prevailing in all 12a-16a structure. Specifically, 12a has a hexa-capped trigonal prism structure with $C_{2 v}$ symmetry and can be constructed by adding two capping atoms to the global-minimum geometry of $\mathrm{Ge}_{10}$ (a tetracapped trigonal prism). Similar global-minimum structure was also obtained previously via tight-binding calculations. ${ }^{34}$ Note that the global-minimum structure of $\mathrm{Si}_{12}$ is the same as 12a (see Fig. 3). The 13a structure has $C_{s}$ symmetry. Again, the lowest-energy structure of $\mathrm{Si}_{13}$ has the same structure as 13a. The global-minimum structure of $\mathrm{Ge}_{14}$ (14a) can be viewed as adding one atom to 13a at the edge of the trigonal prism, while that of $\mathrm{Ge}_{16}$, namely $\mathbf{1 6 a}$ can be viewed as adding two atoms to 14a. However, the global minimum as well as other top-five lowest-energy clusters of $\mathrm{Ge}_{15}(\mathbf{1 5 a} \mathbf{a}$ 15e) appear to follow somewhat different growth pattern from 13a, 14a, and 16a, even though 15a-15e also contain the TTP motif. Here, the cluster growth is along the axial

TABLE II. The binding (or cohesive) energies per atom calculated at the PBEPBE/6-311G $(d)$ level for the low-lying isomers of $\mathrm{Ge}_{12}-\mathrm{Ge}_{20}$ (shown in Fig. 2). Zero-point energy corrections are included in the calculation of the binding energies. The experimental values are taken from Ref. 10.

\begin{tabular}{cccc}
\hline \hline Cluster & Point group & EXPERIMENT $(\mathrm{eV})$ & PBEPBE $/ 6-311 \mathrm{G}(d)(\mathrm{eV})$ \\
\hline $\mathbf{1 2 a}$ & $\boldsymbol{C}_{2 v}$ & 3.210 & $\mathbf{3 . 2 4 5}$ \\
$\mathbf{1 3 a}$ & $\boldsymbol{C}_{s}$ & 3.120 & $\mathbf{3 . 2 2 9}$ \\
$\mathbf{1 4 a}$ & $\boldsymbol{C}_{1}$ & 3.140 & $\mathbf{3 . 2 9 3}$ \\
$\mathbf{1 5 a}$ & $\boldsymbol{C}_{s}$ & 3.150 & $\mathbf{3 . 2 9 7}$ \\
$\mathbf{1 6 a}$ & $\boldsymbol{C}_{2 v}$ & 3.170 & $\mathbf{3 . 2 9 3}$ \\
$\mathbf{1 7 a}$ & $\boldsymbol{C}_{s}$ & 3.150 & $\mathbf{3 . 2 9 8}$ \\
$17 \mathrm{~b}$ & $C_{s}$ & & 3.296 \\
$\mathbf{1 8 a}$ & $C_{3 v}$ & 3.150 & $\mathbf{3 . 3 0 1}$ \\
$18 \mathrm{~b}$ & $C_{s}$ & & 3.285 \\
$\mathbf{1 9 a}$ & $C_{1}$ & 3.150 & $\mathbf{3 . 3 0 9}$ \\
$19 \mathrm{~b}$ & $C_{1}$ & & 3.305 \\
$\mathbf{2 0 a}$ & $C_{2 h}$ & & $\mathbf{3 . 3 2 5}$ \\
$20 \mathrm{~b}$ & $C_{3 v}$ & & 3.311 \\
$20 \mathrm{c}$ & $C_{2 v}$ & & 3.309 \\
\hline \hline
\end{tabular}

direction of the trigonal prism. For $\mathrm{Ge}_{15}$, Ho and co-workers reported two degenerate lowest-energy isomers. ${ }^{37}$ The two isomers differ slightly in the bonding pattern but both have the same point group $C_{s}$. The $15 \mathbf{a}$ is similar to their $C_{s}$ (II) isomer, whereas $15 \mathrm{c}$ and $\mathbf{1 5 d}$ are similar to $C_{s}(\mathrm{I})$. Finally, we note that starting from $n=14$ the global minima of $\mathrm{Ge}_{n}$ are no longer the same as the silicon counterparts (see Fig. 3).

\section{B. $\mathrm{Ge}_{17}-\mathrm{Ge}_{20}$}

The 17a is the lowest-energy isomer based on the biased search with the TTP motif as the seed. We also attempted an unbiased search (using less than $1000 \mathrm{MC}$ trial moves), starting with a random configuration for the initial isomeric structure. That search yielded isomer $\mathbf{1 7} \mathbf{b}$, which is a local minimum but nonetheless also contains the TTP motif and has energy very close to $\mathbf{1 7 a}$ (since the difference in binding energy per atom is less than $5 \mathrm{meV}, \mathbf{1 7 a}$ and $\mathbf{1 7 b}$ may be considered as isoenergetic). Interestingly, on the growth pattern, 17a can be viewed as adding two atoms to $\mathbf{1 5 a}$, whereas 17b can be viewed as adding one atom to 16a. Both 17a and 17b are markedly lower in energy than the global minimum predicted based on tight-binding model. ${ }^{38}$ The 18a was actually obtained solely based on an unbiased search starting from several unrelated isomeric structures. When the TTP motif was used as the seed for the biased search, we also attained the identical isomer 18a. On the growth pattern, the 18a can be viewed as adding one atom to $17 \mathbf{a}$. We also performed a biased search based on the six/six structural motif, which yields the lowest-energy isomer 18b. However, 18b has slightly higher energy than 18a, confirmed by both PBEPBE and B3LYP all-electron DFT calculations. Finally, for the two largest clusters $\mathrm{Ge}_{19}$ and $\mathrm{Ge}_{20}$ considered here, the unbiased BH-DFT search for the global minima becomes extremely computationally demanding (may require up to 10000 MC moves that are beyond our current computing capability). We therefore only carried out three biased searches using three different seeds as mentioned earlier. In contrast to $\mathrm{Ge}_{18}$, the lowest-energy isomer 19a (obtained based on the six/six motif) is slightly lower in energy than the TTP-motif based isomer $19 \mathrm{~b}$. Note that $19 \mathrm{~b}$ can be also obtained via a biased search using the magic-number cluster $\mathrm{Ge}_{10}$ as the seed. Indeed, 19a can be viewed as magicnumber $\mathrm{Ge}_{10}$ plus TTP $\mathrm{Ge}_{9}$. The 19a may also be viewed as adding one atom to $\mathbf{1 8 b}$, whereas $19 \mathbf{b}$ as adding one atom to 18a. In the case of $\mathrm{Ge}_{20}$ the magic-number $\mathrm{Ge}_{10}$ based isomer 20a is the leading candidate for the global minimum. The other two isomers, 20b and 20c, which were obtained based on the six/six motif and the TTP motif, respectively. The 20b is nearly degenerate in energy with 20c.

Finally, we remark that the PBE and B3LYP DFT methods are two very popular choices by many workers to determine energy orderings of medium-sized silicon or germanium clusters. ${ }^{13-15,22-38}$ Hence, the fact that both PBE and B3LYP methods give consistent energy orderings (Table I) among the top-two or top-three low-lying isomers of germanium clusters is very encouraging. For $\mathrm{Ge}_{18}$, in particular, this consistency indicates that 18a is very likely the true global minimum, regardless of DFT method selected. 


\section{Comparison with silicon counterparts}

In the size range of $12 \leqslant n \leqslant 20$, both silicon and the germanium clusters show prolate geometry. Their growth patterns diverge at $n=13$ as predicted earlier. ${ }^{37}$ For lowestenergy silicon clusters, we have recently shown that the TTP-to-six/six motif transition is likely to occur at $n=16,{ }^{31}$ and that for $16 \leqslant n \leqslant 22$ the global minima of $\mathrm{Si}_{n}$ all contain the six/six motif. In contrast, for lowest-energy germanium clusters, the TTP-to-six/six motif transition may occur at $n$ $=19$, and at $n=20$ the magic-number cluster $\mathrm{Ge}_{10}$ appears to be a more preferred structural motif.

\section{CONCLUSIONS}

By means of the BH-DFT approach, we have performed an unbiased search for the global-minimum isomeric structures of germanium clusters $\mathrm{Ge}_{12}-\mathrm{Ge}_{18}$, and a biased search based on three structural motifs (each as a seed) for $\mathrm{Ge}_{17}-\mathrm{Ge}_{20}$. All low-lying clusters in this size range show prolate geometry. In contrast, smaller germanium clusters $(8 \leqslant n \leqslant 11)$ are all compact and spherical-like in shape. Except $\mathrm{Ge}_{19}$, the predicted global-minimum structures all contain the TTP structural motif. In addition, the sixfold puckered ring subunit $\mathrm{Ge}_{6}$ appears in many low-lying isomers, e.g., 17a, 18a, 18b, and 20b. Compared to the predicted global minima of medium-sized silicon clusters in the range of $12 \leqslant n \leqslant 20$, the TTP-to-six/six motif transition for germanium clusters is likely to occur at a size larger than $n=16 .{ }^{40}$

\section{ACKNOWLEDGMENTS}

We thank Dr. J.L. Wang and Dr. J.J. Zhao for valuable discussions. The work was supported by grants from the National Science Foundation (CHE, DMII, and MRSEC), DOE's Office of Basic Energy Sciences (DE-FG0204ER46164), the John Simon Guggenheim Foundation, and the Nebraska Research Initiatives. Computing time was provided by the Research Computing Facility and Bioinformatics Facility at University of Nebraska-Lincoln.

${ }^{1}$ W. L. Brown, R. R. Freeman, K. Raghavachari, and M. Schluter, Science 235, 860 (1987)

${ }^{2}$ Y. Liu, Q.-L. Zhang, F. K. Tittel, R. F. Curl, and R. E. Smalley, J. Chem. Phys. 85, 7434 (1986); J. L. Elkind, J. M. Alford, F. D. Weiss, R. T. Laaksonene, and R. E. Smalley, ibid. 87, 2397 (1987); Q. L. Zhang, Y. Liu, R. F. Curl, F. K. Tittel, and R. E. Smalley, ibid. 88, 1670 (1988).

${ }^{3}$ M. F. Jarrold, Science 252, 1085 (1991); M. F. Jarrold, and V. A. Constant, Phys. Rev. Lett. 67, 2994 (1991); M. F. Jarrold and J. E. Bower, J. Chem. Phys. 96, 9180 (1992).

${ }^{4}$ R. R. Hudgins, M. Imai, M. F. Jarrold, and P. Dugourd, J. Chem. Phys. 111, 7865 (1999); A. A. Shvartsburg, R. R. Hudgins, P. Dugourd, and M. F. Jarrold, Chem. Soc. Rev. 30, 36 (2001).

${ }^{5}$ K.-D. Rinnen and M. L. Mandich, Phys. Rev. Lett. 69, 1823 (1992).
${ }^{6}$ K. Fuke, K. Tsukamoto, F. Misaizu, and M. Sanekata, J. Chem. Phys. 99, 7807 (1993).

${ }^{7}$ S. Hayashi, Y. Kanzawa, M. Kataoka, T. Nagarede, and K. Yamamoto, Z. Phys. D: At., Mol. Clusters 26, 144 (1993).

${ }^{8}$ S. Li, R. J. Van Zee, W. Weltner, Jr., and K. Raghavachari, Chem. Phys. Lett. 243, 275 (1995).

${ }^{9}$ S. Yoshida and K. Fuke, J. Chem. Phys. 111, 3880 (1999).

${ }^{10}$ J. M. Hunter, J. L. Fye, M. F. Jarrold, and J. E. Bowers, Phys. Rev. Lett. 73, 2063 (1994).

${ }^{11}$ D. E. Bergeron and A. W. Castleman, Jr., J. Chem. Phys. 117, 3219 (2002).

${ }^{12}$ B. Marsen, M. Lonfat, P. Scheier, and K. Sattler, Phys. Rev. B 62, 6892 (2000).

${ }^{13}$ K.-M. Ho, A. A. Shvartsburg, B. Pan, Z.-Y. Lu, C.-Z. Wang, J. G. Wacker, J. L. Fye, and M. F. Jarrold, Nature (London) 392, 582 (1998).

${ }^{14}$ A. Bahel and M. V. Ramakrishna, Phys. Rev. B 51, 13849 (1995); M. V. Ramakrishna and A. Bahel, Chem. Phys. 104, 9833 (1996).

${ }^{15}$ Z. Y. Lu, C. Z. Wang, and K.-M. Ho, Phys. Rev. B 61, 2329 (2000).

${ }^{16}$ L. Mitas, J. C. Grossman, I. Stich, and J. Tobik, Phys. Rev. Lett. 84, 1479 (2000).

${ }^{17}$ J. R. Chelikowsky and J. C Phillips, Phys. Rev. Lett. 63, 1653 (1989)

${ }^{18}$ E. Kaxiras, Phys. Rev. Lett. 64, 551 (1990).

${ }^{19}$ D. A. Jelski, B. L. Swift, T. T. Rantala, X. Xia, and T. F. George, J. Chem. Phys. 95, 8552 (1991).

${ }^{20}$ U. Röthlisberger, W. Andreoni, and M. Parrinello, Phys. Rev. Lett. 72, 665 (1994).

${ }^{21}$ M. Menon and K. R. Subbaswamy, Phys. Rev. B 51, 17952 (1995).

${ }^{22}$ M. R. Pederson, K. Jackson, D. V. Porezag, Z. Hajnal, and Th. Frauenheim, Phys. Rev. B 54, 2863 (1996).

${ }^{23}$ B. X. Li, P. L. Cao, and S.-C. Zhan, Phys. Lett. A 316, 252 (2003).

${ }^{24}$ A. Sieck, Th. Frauenheim, and K. A. Jackson, Phys. Status Solidi B 240, 537 (2003).

${ }^{25}$ Q. Sun, Q. Wang, P. Jena, S. Waterman, and Y. Kawazoe, Phys. Rev. A 67, 063201 (2003).

${ }^{26}$ I. Rata, A. A. Shvartsburg, M. Horoi, Th. Frauenheim, K. W. M. Siu, and K. A. Jackson, Phys. Rev. Lett. 85, 546 (2000).

${ }^{27}$ S. Yoo, X. C. Zeng, X. Zhu, and J. Bai, J. Am. Chem. Soc. 125, 13318 (2003).

${ }^{28}$ A. Tekin and B. Hartke, Phys. Chem. Chem. Phys. 6, 503 (2004).

${ }^{29}$ K. A. Jackson, M. Horoi, I. Chaudhuri, T. Frauenheim, and A. A. Shvartsburg, Phys. Rev. Lett. 93, 013401 (2004).

${ }^{30}$ S. Yoo, J. Zhao, J. Wang, and X. C. Zeng, J. Am. Chem. Soc. 126, 13845 (2004).

${ }^{31}$ S. Yoo and X. C. Zeng, Angew. Chem., Int. Ed. 44, 1491 (2005).

${ }^{32}$ S. Orgut and J. R. Chelikowsky, Phys. Rev. B 55, R4914 (1997).

${ }^{33}$ P. W. Deutsch, L. A. Curtiss, and J. P. Blaudeau, Chem. Phys. Lett. 270, 413 (1997).

${ }^{34}$ E. F. Archibong and A. St-Amant, J. Chem. Phys. 109, 962 (1998).

${ }^{35}$ B. X. Li and P. L. Cao, Phys. Status Solidi B 219, 253 (2000).

${ }^{36}$ Z.-Y. Lu, C. Z. Wang, and K.-M. Ho, Phys. Rev. B 61, 2329 (2000).

${ }^{37}$ A. A. Shvartsburg, B. L., Z.-Y. Lu, C.-Z. Wang, M. F. Jarrold, and K. M. Ho, Phys. Rev. Lett. 83, 2167 (1999).

${ }^{38}$ J. Wang, G. H. Wang, and J. Zhao, Phys. Rev. B 64, 205411 (2001).

${ }^{39}$ D. J. Wales, M. A. Miller, and T. R. Walsh, Nature (London) 394, 758 (1998).

${ }^{40}$ D. J. Wales and H. A. Scheraga, Science 285, 1368 (1999).

${ }^{41}$ R. Car and M. Parrinello, Phys. Rev. Lett. 55, 2471 (1985).

${ }^{42}$ J. Hutter, A. Alavi, T. Deutsch, M. Bernasconi, S. Goedecker, D. Marx, M. Tuckerman, and M. Parrinello, CPMD, V3.7.1, MPI für Festkörperforschung, Stuttgart, 1997-2001.

${ }^{43}$ W. Kohn and L. J. Sham, Phys. Rev. 140, A1133 (1965).

${ }^{44}$ M. J. Frisch et al., GAUSSIAN 03, Revision C.02, Gaussian, Inc., Wallingford (2004). 